\title{
Pacientes como informadores de eventos adversos. Resultados en diabetes y enfermedad renal
}

\author{
Patients informing of adverse events. Results in diabetes \\ and kidney disease
}

\author{
JJ. Mira ${ }^{1,2,3}$, J. Vitaller ${ }^{1,2,4}$, S. Lorenzo ${ }^{5}$, C. Royuela ${ }^{5}$, V. Pérez-Jover ${ }^{1}$, J. Aranaz ${ }^{1,2,6}$
}

\section{RESUMEN}

Fundamento. Diabetes y enfermedad renal son factores de riesgo de sufrir eventos adversos (EA). No contamos con estudios sobre la percepción del riesgo de estos pacientes. En este estudio se analiza la frecuencia con la que los pacientes diabéticos y renales describen indicios de un posible EA y su percepción de seguridad de la atención que reciben.

Material y métodos. Estudio descriptivo basado en entrevistas a pacientes seleccionados al azar. Estudio de campo realizado entre febrero y mayo de 2010 en 3 centros de salud y 2 hospitales de Alicante y Madrid.

Resultados. Respondieron 199 pacientes, 98 diabéticos y 101 con enfermedad renal. Estos últimos acumularon mayor número de indicios de EA (21,8\% refirió un EA, 17,8\% dos y un $3 \% 3$ o más) que los diabéticos (16,3\% un EA, $7,1 \%$ dos y $7,1 \%$ señaló 3 o más). En el último año, 6/98 diabéticos y $10 / 101$ enfermos renales precisaron un tratamiento adicional. La probabilidad que el paciente cree tener de ser víctima de un error con consecuencias graves fue establecida en 1:10. Las mujeres con enfermedad renal creyeron tener una mayor probabilidad de sufrir un error $\left(\mathrm{Chi}^{2}=12,7, \mathrm{p}=0,002\right)$. Los errores clínicos se atribuyeron a la falta de tiempo para atender a todos los pacientes y a la insuficiencia de medios y recursos, sin diferencias estadísticamente significativas entre las submuestras. Los pacientes entrevistados consideraron similar el riesgo de error clínico, accidente de tráfico, atraco.

Conclusiones. La información que proporcionan los pacientes puede contribuir a mejorar la seguridad de los procedimientos de trabajo.

Palabras clave. Seguridad clínica. Pacientes diabéticos. Pacientes renales. Percepción.

\begin{abstract}
Background. Diabetes and kidney disease are risk factors for adverse events (AE). There are no other studies on the perception of risk in these patients. This study analyzes the frequency of adverse event triggers reported by diabetic and renal patients and their perception of the risk
\end{abstract}

Material and methods. Descriptive study based on interviews with randomly selected patients. Field study conducted between February and May 2010 in three health centers and two hospitals in Alicante and Madrid.

Results. A total of 199 patients answered, 98 diabetic patients and 101 renal patients. Renal patients accumulated more $\mathrm{AE}$ triggers (21.8\% referred to an $\mathrm{AE}$ trigger, $17.8 \%$ two $\mathrm{AE}$ triggers and $3 \%$ referred to $>3 \mathrm{AE}$ triggers) than diabetic patients (16.3\% referred to one $\mathrm{AE}$ trigger, $7.1 \%$ to two $\mathrm{AE}$ triggers and $7.1 \%$ referred to $>3 \mathrm{AE}$ triggers). During the last year 6/98 diabetic patients and 10/101 renal patients required additional treatment due to a clinical error. The probability of the patient being the victim of a clinical error with serious consequences was 1:10. Women with renal illness believed themselves to have a greater probability of suffering an error $\left(\mathrm{Chi}^{2}=12.7, \mathrm{p}=0.002\right)$. Errors were attributed to a lack of time to attend to all patients and a lack of means and resources, without statistically significant differences between the subsamples. Interviewed patients considered that the risks of suffering a traffic accident or robbery were similar to the risk of an error with serious consequences.

Conclusion. Information provided by patients can help improve safety procedures.

Key words. Safety. Diabetes complications. Kidney diseases. Perception.
1. Universidad Miguel Hernández. Elche

2. CIBER Epidemiología y Salud Pública (CIBERESP)

3. Departamento de Salud 17. Consellería Sanitat. Generalitat Valenciana. Alicante

4. Inspección Médica. Consellería Sanitat. Generalitat Valenciana. Elche

5. Hospital Universitario Fundación Alcorcón. Alcorcón, Madrid

6. Hospital General Universitario de Sant Joan. Alicante

Recepción: 18 de noviembre de 2011

Aceptación provisional: 12 de enero de 2012

Aceptación definitiva: 3 de febrero de 2012

\section{Correspondencia:}

José Joaquín Mira Solves

Universidad Miguel Hernández

Avda. de la Universidad $\mathbf{s} / \mathrm{n}$

03202 Elche

E-mail: jose.mira@umh.es

Este estudio forma parte de un proyecto sobre errores de pacientes financiado por el Fondo de Investigación Sanitaria y cuenta también con financiación de fondos FEDER, referencia PI08-90118. 


\section{INTRODUCCIÓN}

El diseño de los estudios en los que se intenta determinar la frecuencia con la que los pacientes sufren un evento adverso (EA) ha ido variando a lo largo del tiempo. Primero, se basaron en diseños retrospectivos, a partir de documentación clínica del paciente, posteriormente en estudios prospectivos y, más recientemente, en los resultados de encuestas a pacientes.

La frecuencia con la que pacientes ingresados sufren un EA oscila, según los estudios retrospectivos, entre un $4^{1} \mathrm{y}$ un $17 \%^{2}$ y alcanza el $31 \%^{3}$ en estudios prospectivos. Las reacciones a los medicamentos son, por lo que sabemos, el EA más frecuente (19\%), seguido de las infecciones nosocomiales de herida quirúrgica $(14 \%)^{1,4}$. Un $50 \%$ de estos EA podrían haberse evitado según estos estudios ${ }^{2}$. En diálisis, hasta 9 de cada 100 pacientes sufren un EA mensualmente ${ }^{5}$. En el caso de EA en atención primaria existe mayor variabilidad y las cifras de frecuencia de EA oscilan entre el 2 y el $76 \%$ del total de consultas ${ }^{6,7}$. La mitad de estos EA están relacionados con la medicación ${ }^{8,9}$. En un reciente estudio prospectivo realizado con pacientes diabéticos tipo 2 , la actuación profesional estuvo en la génesis de un $6 \%$ de los EA y los problemas de comunicación médico-paciente ocasionaron un $59 \%{ }^{10}$ de los EA identificados.

En las encuestas a pacientes se observa también variabilidad en las cifras ${ }^{11}$, oscilando la frecuencia de EA cuando se pregunta por hospitalizaciones recientes entre un 6 y un $13 \%{ }^{12,13}$. En estas cifras influye el tipo de pregunta que se formula ${ }^{14}$. En atención primaria en España, un 6,2\% dijo haber sufrido un $\mathrm{EA}^{15}$ y un $17,6 \%$ de los pacientes adultos y el $13,7 \%$ de los pediátricos solicitó segunda consulta por complicaciones o reacciones inesperadas con su tratamiento $^{16}$. En dos estudios sucesivos realizados en Adelaida ${ }^{17}$ (Australia) un 4,2 y un 6,5\% de los pacientes, respectivamente, informaron haber sufrido un EA que les había ocasionado algún daño o lesión.
Preguntar directamente a los pacientes ha generado algunas dudas sobre la fiabilidad de la información obtenida por este método $^{18}$. Sin embargo, nuevos estudios han puesto de manifiesto que los pacientes pueden ser un informador fiable ${ }^{19}$, especialmente cuando el EA tiene consecuencias más graves ${ }^{20}$.

La implicación del paciente en la seguridad se ha subrayado como una necesidad de la práctica clínica ${ }^{21}$, para lo que es necesario que el paciente sea consciente de los riesgos inherentes de práctica clínica. Este punto es particularmente importante en nuestro caso, porque según datos recientes, menos de la mitad de los españoles espera que se produzcan errores clínicos ${ }^{22}$ y la mayoría considera que es improbable ser víctima de un error durante una estancia hospitalaria ${ }^{23}$.

En España, los estudios ENEAS ${ }^{4}$ y APEAS $^{9}$ sugieren que diabetes y enfermedad renal constituyen dos de los factores de riesgo intrínseco más importantes para sufrir EA. También son los pacientes con mayor riesgo de sufrir un EA relacionado con la medicación con consecuencias graves $^{24}$. Sin embargo, la perspectiva de los pacientes no ha sido estudiada y apenas contamos con estudios sobre la percepción del riesgo de sufrir un EA de estos pacientes. En este estudio se analiza la frecuencia de EA experimentados por pacientes diabéticos y renales y su percepción del riesgo, en base a la información que proporcionan los propios pacientes.

\section{MATERIAL Y MÉTODOS}

Estudio descriptivo basado en entrevistas a pacientes diabéticos y renales en tratamiento en centros de salud o unidad de diálisis. El estudio de campo se realizó entre febrero y mayo de 2010 en tres centros de salud y dos hospitales (en consultas externas) de las provincias de Alicante y Madrid. El estudio fue aprobado por el Comité de Ética de la Universidad Miguel Hernández. 
El guión de entrevista (ver Anexo 1) se diseñó a partir de las aportaciones de pacientes y profesionales en un estudio cualitativo realizado un año antes ${ }^{13,23}$. A los pacientes se les preguntó por síntomas asociados con frecuencia a EA según el estudio APEAS (lo que consideramos como indicios de haber sufrido un EA). En concreto, se preguntó si en el curso del tratamiento había padecido alguna reacción inadecuada a algún medicamento, como prurito, erupción, picor, calambres; si había tenido alguna reacción alérgica; o si había precisado ingresar en el hospital por alguna complicación inesperada con el tratamiento. Adicionalmente, con respecto al último año de tratamiento, se les preguntó si habían necesitado un tratamiento adicional, incluido ingreso hospitalario, por lo que consideraban un error en la atención recibida. Se aplicaron también las subescalas de confianza, percepción de riesgos y causas probables de los errores clínicos, del Cuestionario de Percepción de Seguri$\mathrm{dad}^{23}$. Por último, se preguntó por la satisfacción con la asistencia recibida mediante una pregunta directa. El procedimiento del estudio no vinculaba ningún dato de carácter personal con las respuestas. Para participar en este estudio se aplicó un protocolo de información y obtención de consentimiento. Los coordinadores de los centros de salud, los médicos de las consultas de los centros de especialidades y la responsable de la unidad de diálisis dieron su consentimiento y facilitaron las entrevistas a los pacientes.

El tamaño muestral fue calculado para un error máximo del $6 \%$ y una $\mathrm{p}=\mathrm{q}=0,5$ y un alfa del 0,05 (unilateral). La selección de pacientes se realizó eligiendo al azar días de la semana para acudir a los centros sanitarios (centros de salud y centros de especialidades) a realizar las entrevistas a los pacientes con consulta ese día y que cumplieran los criterios de inclusión: mayor de edad, curso de al menos 3 años y capaci- dad para responder. En caso de que el paciente rechazara contestar, se le sustituía por otro paciente que hubiera acudido ese mismo día a consulta, reclutado también aleatoriamente, procediendo de este modo hasta completar el tamaño muestral. El nivel de rechazo a responder no superó el $5 \%$. Los datos los recogieron tres entrevistadores experimentados (dos enfermeras y una titulada en ciencias) que realizaron varias entrevistas previas para unificar criterios de codificación y estilo al formular las preguntas.

Antes del análisis de los datos se verificó la calidad de los registros, comprobando que no existían errores de codificación.

El análisis estadístico se realizó mediante el estadístico Chi Cuadrado, considerando de forma convencional que existían diferencias estadísticamente significativas cuando $\mathrm{p}<0,05$.

\section{RESULTADOS}

Respondieron en el plazo indicado 199 pacientes, 98 diabéticos y 101 enfermos renales (Tabla 1). Los enfermos renales acumularon un mayor número de indicios de $\mathrm{EA}$ en el curso de su tratamiento (el 21,8\% dijo haber experimentado uno de los signos considerado indicio de EA, el 17,8\% dos indicios y un $3 \%$ aseguró haber padecido 3 o más de estos indicios) que los diabéticos (16,3\% dijo haber experimentado uno, el 7,1\% dos y un $7,1 \%$ señaló 3 o más de estos indicios). La frecuencia con la que cada submuestra informó de algún indicio de haber sufrido un EA en el curso de su tratamiento en el último año se describe en la tabla 2.

En el último año, 6/98 diabéticos y $10 / 101$ enfermos renales precisaron un tratamiento adicional a raíz de lo que consideraban un error clínico. En 3 y 7 casos, respectivamente, precisaron ingreso hospitalario por esta razón, en este caso el rango de la estancia osciló entre 1 y 30 días (mediana 2) (Tabla 2). 
Tabla 1. Características de la muestra de pacientes con diabetes o enfermedad renal

\begin{tabular}{lcc}
\hline & $\begin{array}{c}\text { Diabetes } \\
(\mathbf{N}=98)\end{array}$ & $\begin{array}{c}\text { Enfermedad renal } \\
(\mathbf{N}=\mathbf{1 0 1})\end{array}$ \\
\hline Edad* $^{*}$ & $54,1(20,7)$ & $61,7(15,6)$ \\
Años en tratamiento* & $14,4(11,3)$ & $4,8(6,1)$ \\
& $\mathrm{N}(\%)$ & $\mathrm{N}(\%)$ \\
\hline
\end{tabular}

\begin{tabular}{lcc}
\hline $\begin{array}{l}\text { Género } \\
\text { Mujeres }\end{array}$ & $50(51 \%)$ & $51(50,5 \%)$ \\
\hline Estado civil & & \\
Soltero & $24(24,5 \%)$ & $17(16,8 \%)$ \\
Casado & $47(48,0 \%)$ & $63(63,4 \%)$ \\
Viudo & $22(22,4 \%)$ & $14(13,9 \%)$ \\
$\quad$ Divorciado & $5(5,1 \%)$ & $7(6,9 \%)$ \\
\hline Estudios & & \\
Básicos & $60(61,2 \%)$ & $74(73,3 \%)$ \\
$\quad$ Form. profesional & $24(24,5 \%)$ & $17(16,7 \%)$ \\
$\quad$ Universitarios & $14(14,3 \%)$ & $10(10,0 \%)$ \\
\hline *Media (dt) & &
\end{tabular}

Tabla 2. Frecuencia con la que los pacientes informan haber experimentado alguno de los siguientes indicios de un EA

\begin{tabular}{lccc}
\hline & $\begin{array}{c}\text { Total } \\
(\mathbf{N = 1 9 9 )}\end{array}$ & $\begin{array}{c}\text { Diabetes } \\
\text { (N=98) }\end{array}$ & $\begin{array}{c}\text { Enfermedad renal } \\
\text { (N=101) }\end{array}$ \\
\hline $\begin{array}{l}\text { En el curso del tratamiento ha sufrido alguna reacción inadecuada } \\
\text { a algún medicamento (prurito, erupción, picor, calambres) }\end{array}$ & $51(25,6)$ & $16(16,3)$ & $35(34,7)$ \\
\hline En el curso del tratamiento ha sufrido alguna reacción alérgica & $30(15,1)$ & $10(10,2)$ & $20(19,8)$ \\
\hline $\begin{array}{l}\text { En el curso del tratamiento ha precisado ingresar en el hospital } \\
\text { por alguna complicación inesperada con el tratamiento }\end{array}$ & $25(12,6)$ & $13(13,3)$ & $12(11,9)$ \\
\hline $\begin{array}{l}\text { Ha precisado de un tratamiento adicional por lo que considera } \\
\text { un error clínico }\end{array}$ & $6(3)$ & $3(3,1)$ & $3(3)$ \\
\hline $\begin{array}{l}\text { Ha precisado hospitalización por lo que considera un error en } \\
\text { el tratamiento }\end{array}$ & $10(5)$ & $3(3,1)$ & $7(6,9)$ \\
\hline
\end{tabular}

La probabilidad para el paciente de ser víctima de un error clínico con consecuencias graves fue establecida en 1:10 (23/199, probabilidad estimada del $11,6 \%$ ) (Tabla 3). Ni el estado civil $\left(\mathrm{Chi}^{2}=2,2 ; \mathrm{p}=0,09\right)$, ni el nivel de estudios concluidos $\left(\mathrm{Chi}^{2}=5,6\right.$; $\mathrm{p}=0,23$ ) modificaron esta percepción. Exis- tió efecto cruzado de las variables sexo y patología, de tal modo que las mujeres con enfermedad renal creyeron tener una mayor probabilidad de ser víctima de un error clínico en el curso de su tratamiento $\left(\mathrm{Chi}^{2}=12,7 ; \mathrm{p}=0,002\right)$. 
Tabla 3. Probabilidad que el paciente cree tener de sufrir un error clínico con consecuencias graves

\begin{tabular}{lccc}
\hline & $\begin{array}{c}\text { Total } \\
(\mathbf{N = 1 9 9 )}\end{array}$ & $\begin{array}{c}\text { Diabetes } \\
(\mathbf{N}=\mathbf{9 8})\end{array}$ & $\begin{array}{c}\text { Enfermedad renal } \\
(\mathbf{N}=\mathbf{1 0 1})\end{array}$ \\
\hline Baja & $125(62,8)$ & $57(58,2)$ & $68(67,3)$ \\
Media & $51(25,6)$ & $28(28,6)$ & $23(22,8)$ \\
Alta & $23(11,6)$ & $13(13,3)$ & $10(9,9)$ \\
\hline
\end{tabular}

Pregunta extraída del Cuestionario de Percepción de Seguridad ${ }^{23}$

Entre paréntesis, datos en \%

El hecho de haber informado de una reacción inadecuada a la medicación $\left(\mathrm{Chi}^{2}=4,6 ; \mathrm{p}=0,1\right)$, precisar ingreso $\left(\mathrm{Chi}^{2}=4,9 ; \mathrm{p}=0,09\right)$ o un tratamiento adicional $\left(\mathrm{Chi}^{2}=0,6, \mathrm{p}=0,7\right)$ a raíz de un error clínico, no afectó a la percepción de pro- babilidad de riesgo de sufrir error clínico. Los pacientes entrevistados consideraron similar el riesgo de accidente de tráfico, de sufrir atraco o robo, o de experimentar un error clínico con consecuencias graves (Tabla 4).

Tabla 4. Probabilidad de riesgos de la vida cotidiana, según informe del paciente

\begin{tabular}{lccc}
\hline & $\begin{array}{c}\text { Total } \\
(\mathbf{N}=\mathbf{1 9 9})\end{array}$ & $\begin{array}{c}\text { Diabetes } \\
(\mathbf{N}=\mathbf{9 8})\end{array}$ & $\begin{array}{c}\text { Enfermedad renal } \\
(\mathbf{N}=\mathbf{1 0 1})\end{array}$ \\
\hline Cortocircuito en hogar & $8(4)$ & $3(3,1)$ & $5(5,0)$ \\
\hline Atraco/robo & $22(11,1)$ & $12(12,2)$ & $10(9,9)$ \\
\hline Accidente de tráfico & $23(11,6)$ & $13(13,3)$ & $10(9,9)$ \\
\hline Error clínico & $23(11,6)$ & $13(13,3)$ & $10(9,9)$ \\
\hline
\end{tabular}

Pregunta extraída del Cuestionario de Percepción de Seguridad ${ }^{23}$. Entre paréntesis, datos en \%

La confianza en médicos y enfermeros fue alta, aunque fue mayor entre los enfermos renales (Tabla 5). Esta confianza se mantuvo con independencia del sexo, estado civil o estudios concluidos.

Tabla 5. Confianza manifestada en unidades y profesionales

\begin{tabular}{lccccc}
\hline & $\begin{array}{c}\text { Total } \\
(\mathbf{N}=199)\end{array}$ & $\begin{array}{c}\text { Diabetes } \\
(\mathbf{N}=\mathbf{9 8})\end{array}$ & $\begin{array}{c}\text { Enfermedad renal } \\
(\mathbf{N = 1 0 1 )}\end{array}$ & Chi $^{\mathbf{2}}$ & $\mathbf{p =}$ \\
\hline $\begin{array}{l}\text { Unidad/Centro/Consulta en } \\
\text { la que está siendo tratado }\end{array}$ & $166(83)$ & $78(78,8)$ & $88(87,1)$ & 7,8 & 0,02 \\
$\quad$ Médicos & $163(81,5)$ & $75(75,8)$ & $88(87,1)$ & 8,8 & 0,01 \\
$\quad$ Enfermeros & $158(79)$ & $71(71,7)$ & $87(86,1)$ & 8,1 & 0,02 \\
\hline
\end{tabular}

Pregunta extraída del Cuestionario de Percepción de Seguridad ${ }^{23}$. Sólo se incluyen los pacientes con un alto nivel de confianza en Unidades/Centro/Consulta, médicos o enfermeros. Entre paréntesis, datos en \%.

Los pacientes que informaron de su ingreso en el hospital a raíz de un error, manifestaron una pérdida de confianza en la unidad asistencial de 17 puntos porcentua- les de media (de forma más acusada en el caso de enfermos en diálisis, 25 puntos porcentuales) $\left(\mathrm{Chi}^{2}=5,6 ; \mathrm{p}=0,02\right)$. Cuando informaron que habían precisado un tratamiento 
adicional a raíz de un error clínico en el último año, la confianza en el médico bajó una media de 15 puntos porcentuales (que llegó hasta 36 puntos porcentuales en el caso de los enfermos renales) $\left(\mathrm{Chi}^{2}=7,9 ; \mathrm{p}=0,0005\right)$. La satisfacción del paciente también disminuyó significativamente $\left(\mathrm{Chi}^{2}=3,8 ; \mathrm{p}=0,005\right)$. La confianza de los pacientes diabéticos en el enfermero que sigue su evolución se vio deteriorada únicamente cuando se informa- ba haber experimentado una reacción alérgica, perdiendo de media hasta 18 puntos porcentuales $\left(\mathrm{Chi}^{2}=5,3: \mathrm{p}=0,02\right)$.

Los supuestos errores clínicos, en opinión de los entrevistados, se debieron sobre todo a la falta de tiempo para atender a todos los pacientes y a la insuficiencia de medios y recursos, sin diferencias estadísticamente significativas entre ambas submuestras (Tabla 6).

Tabla 6. Causas habituales de los errores clínicos a juicio de los pacientes

\begin{tabular}{lccc}
\hline & $\begin{array}{c}\text { Total } \\
(\mathbf{N}=\mathbf{1 9 9})\end{array}$ & $\begin{array}{c}\text { Diabetes } \\
(\mathbf{N}=\mathbf{9 8})\end{array}$ & $\begin{array}{c}\text { Enfermedad renal } \\
(\mathbf{N}=\mathbf{1 0 1})\end{array}$ \\
\hline Escasa formación de los profesionales & $7(3,5)$ & $4(4,1)$ & $3(3,3)$ \\
Desorganización del propio centro & $13(6,5)$ & $8(8,2)$ & $5(5,0)$ \\
Fatiga de los profesionales & $14(7)$ & $8(8,2)$ & $6(5,9)$ \\
Insuficientes medios y recursos & $21(10,6)$ & $13(13,3)$ & $8(7,9)$ \\
$\begin{array}{l}\text { Falta de tiempo para atender a todos } \\
\quad \text { los pacientes }\end{array}$ & $23(11,6)$ & $13(13,3)$ & $10(9,9)$ \\
\hline
\end{tabular}

Pregunta extraída del Cuestionario de Percepción de Seguridad ${ }^{23}$. Sólo se incluyen los pacientes que atribuyeron inequívocamente a estas causas los errores clínicos (opción de respuesta: siempre). Entre paréntesis datos en \%.

\section{DISCUSIÓN}

Los pacientes constituyen una fuente relevante y complementaria de información para mejorar la seguridad clínica. Pueden informar de fallos de sistemas y de errores, en ambos casos información útil para modificar protocolos y guías. También, nos ofrecen pistas sobre su percepción de seguridad (confianza) en el sistema sanitario y sus profesionales, que permite revisar la información que conviene trasladar a los pacientes. En este caso, un 6\% de los diabéticos y un $10 \%$ de los enfermos en diálisis dijeron haber sido víctimas de un error clínico que requirió un tratamiento adicional, incluido el ingreso hospitalario. Estas cifras, son cercanas a las aportadas en estudios prospectivos con revisión de la situación clínica del paciente, tanto en el caso de diabetes ${ }^{10}$, como de diálisis ${ }^{5}$. La distinta metodología empleada en esos estudios no hace factible la comparación.

Estos resultados refuerzan la impresión de que los pacientes pueden contribuir a la mejora de la atención sanitaria, informando de la ocurrencia de incidentes para la seguridad de los pacientes ${ }^{11,14}$. En dos recientes estudios, la fiabilidad de los informes de los pacientes (contrastada con la información clínica disponible) oscilaba entre el 55 y el $77 \%^{19,20}$. Como dato, en el estudio $\mathrm{ENEAS}^{4}$, el número de clasificaciones correctas (acuerdo entre profesionales al revisar la historia clínica en busca de EA) osciló entre $65-80 \%$.

En este estudio se solicitaba a los pacientes que informaran de situaciones concretas que suponían indicios razonables de haber experimentado un $\mathrm{EA}^{4,9}$, en cuyo origen estuviera un fallo del sistema, o una intervención de los profesionales. Se incluyeron aquellas situaciones que en la literatura ${ }^{14,25}$ se asociaban a una mayor fiabilidad de la información del paciente, como son los errores relacionados con la medicación o derivados de una reacción alérgica. Los indicios de EA incluidos en el protocolo de investigación tienen con- 
secuencias más graves y son, por tanto, más fáciles de identificar por los pacientes. Los datos en este estudio de que un $30 \%$ de los diabéticos y un $43 \%$ de los sometidos a diálisis experimenten en el curso del tratamiento algún tipo de EA cabe interpretarlos como la punta del iceberg del total de EA, habida cuenta del enfoque metodológico empleado.

Mientras que la percepción del riesgo de sufrir un EA se mantiene estable aún en aquellos casos que han experimentado daño, la confianza de los pacientes, sobre todo en el médico, se deteriora cuando sufre daño, lesión o complicaciones en el curso de un tratamiento. Este resultado corrobora la tendencia en los resultados apuntados en otros estudios ${ }^{12}$. Dado que la confianza es un factor terapéutico fundamental, estos resultados subrayan la importancia de informar correcta y precozmente a los pacientes que han sufrido un EA a raíz de un error clínico, precisamente para mantener esa confianza ${ }^{13,26}$.

Los entrevistados atribuyen la misma probabilidad a sufrir un atraco o robo, un accidente de tráfico o a ser víctima de un error clínico. Este dato hace diferentes a estos colectivos de pacientes, probablemente por su situación clínica ${ }^{25}$, a otros colectivos que consideran muy improbable sufrir un $\mathrm{EA}^{13}$. Según datos de la Asociación Empresarial del Seguro ${ }^{26}$ (UNESPA), en las provincias de Alicante y Madrid se produce un accidente de tráfico con daño material cada 6 minutos y 55 segundos y cada 1 minuto y 27 segundos, respectivamente; y con daño corporal cada hora, 2 minutos y 15 segundos y 13 minutos y 14 segundos respectivamente. No disponemos de datos para determinar con la misma exactitud la frecuencia en términos similares de EA, pero extrapolando los datos del estudio ENEAS, podemos estimar que en los hospitales españoles se produce un EA cada minuto y 11 segundos. Pese a las diferencias, el hecho de que los pacientes igualen la frecuencia de accidentes de tráfico y de EA hay que considerarla positivamente porque supone una mayor preocupación y, por consiguiente, mayores posibilidades de lograr una mayor implicación de los pacientes en su seguridad, si se incluyera en la agenda pautas sobre de qué debe informarse a los pacientes.

La falta de tiempo y carencia de recursos y medios es, a juicio de los pacientes, donde reside fundamentalmente el origen de los EA; resultado similar este dato al hallado con otros colectivos ${ }^{23,29}$. Este resultado refuerza la idea de que los pacientes tienen plena confianza en la competencia de sus profesionales y, en cierto modo, este resultado es probablemente reflejo de ese rol más pasivo que se observa en nuestras consultas.

No obstante, los resultados deben interpretarse con prudencia. Primero, porque la información que han proporcionado los pacientes no ha sido contrastada con la documentación clínica para preservar el anonimato de pacientes y profesionales, aunque tiene en cuenta los estudios que sugieren que la fiabilidad de estos enfoques es alta ${ }^{18}$, sobre todo en relación a la medicación ${ }^{27}$. Segundo, porque en la redacción de las preguntas se utilizó el término error habida cuenta de los estudios que sugieren que es el término más apropiado para este tipo de consulta $^{23}$, si bien pudiera haber limitado el alcance del informe del paciente que podría suponerse posee una mayor familiaridad con la jerga y condiciones de la práctica clínica. Futuros estudios podrían considerar la influencia en la percepción del riesgo de la comorbilidad frecuente en estos pacientes $\mathrm{u}$ otras variables como los años en tratamiento o la percepción de las personas más mayores, polimedicadas.

La información que proporcionan los pacientes puede contribuir a mejorar la seguridad de los procedimientos de trabajo. Estos resultados sugieren, por un lado, que este tipo de estudios pueden ser un complemento a los estudios epidemiológicos para determinar frecuencia, causas y consecuencias de los EA; por otro, refuerza los enfoques que promueven la participación del paciente en los sistemas de notificación de EA.

\section{Agradecimientos}

Mercedes Guilabert, Isabel María y Alicia Peralta han contribuido en la recolección de 
datos y en la preparación de bases de datos. Jesús Casal y José Antonio Picó revisaron y aportaron ideas para mejorar los instrumentos utilizados. José Francisco Herrero,
Domingo Orozco, Vicente Gil, Lidia Ortiz, Antonio Ochando y Encarnación Hernández han colaborado desinteresadamente en distintos momentos de la investigación.

Anexo 1. Qué puede y qué debe hacer un/a paciente diabético para mejorar la seguridad clínica

\begin{tabular}{|l|c|c|c|}
\hline & SÍ, MUCHO & SÍ, EN PARTE & NO, NADA \\
\hline $\begin{array}{l}\text { 1. ¿Está usted satisfecho/a con el resultado de la atención } \\
\text { sanitaria que está recibiendo? }\end{array}$ & $\square$ & $\square$ \\
\hline
\end{tabular}

2. Indique el grado de confianza (sensación de estar en "buenas manos") que le inspira:

\begin{tabular}{|c|c|c|c|c|}
\hline & & $\begin{array}{c}\text { CONFIANZA } \\
\text { ALTA }\end{array}$ & $\begin{array}{c}\text { CONFIANZA } \\
\text { MEDIA }\end{array}$ & $\begin{array}{c}\text { CONFIANZA } \\
\text { ВАJA }\end{array}$ \\
\hline a- & El centro donde está siendo tratado & & & \\
\hline b- & El médico/a que le atienden & & & \\
\hline$c-$ & El enfermero/a que le atienden & & & \\
\hline d- & Los auxiliares de enfermería que le atienden & & & \\
\hline
\end{tabular}

\begin{tabular}{|l|c|c|c|}
\hline & MUCHO & POCO & NADA \\
\hline $\begin{array}{l}\text { 3. En su caso concreto } \\
\text { clínico preocupa sufrir un error }\end{array}$ & $\square$ & $\square$ & $\square$ \\
\hline
\end{tabular}

\begin{tabular}{|l|c|c|}
\hline 4. En su caso concreto indique si le ha pasado alguna de estas cosas? & SÍ & NO \\
Ingresar en el hospital por alguna complicacióngrave a raíz de un & $\square$ & $\square$ \\
$\quad$ fallo en la atención recibida & $\square$ \\
$\quad \begin{array}{l}\text { Sufrir una reacción alérgicaa un medicamento } \\
\text { Tener una reacción inadecuada a algún medicamento (prurito, } \\
\quad \text { erupción, picor, calambres, vómitos, etc.) }\end{array}$ & $\square$ \\
\hline
\end{tabular}

\begin{tabular}{|c|c|c|c|c|}
\hline \multicolumn{5}{|c|}{ 5. A qué cree usted que se deben sobre todo los errores en el tratamiento de la diabetes: } \\
\hline & & SIEMPRE & A VECES & NUNCA \\
\hline a & Escasa formación de los profesionales sanitarios & & & \\
\hline b & $\begin{array}{l}\text { No disponer de tiempo suficiente para todos los } \\
\text { pacientes }\end{array}$ & & & \\
\hline c & Fatiga o cansancio de los profesionales & & & \\
\hline d & Insuficientes medios o recursos terapéuticos & & & \\
\hline e & Falta de comunicación entre los profesionales & & & \\
\hline $\mathrm{f}$ & Desorganización en el Centro & & & \\
\hline g & $\begin{array}{l}\text { Insuficiente entrenamiento e información al } \\
\text { paciente para que él/ella pueda cuidarse mejor } \\
\text { (autocuidado) }\end{array}$ & & & \\
\hline
\end{tabular}




\begin{tabular}{|l|c|c|c|}
\hline & Sí & NO \\
\hline $\begin{array}{l}\text { 6. En el último año: ¿Ha tenido alguna complicación o ha necesitado un } \\
\text { tratamiento adicional por algún error en la atención que recibe? }\end{array}$ & $\square$ & $\square$ \\
\hline & MUY GRAVES & POCO GRAVES & NADA GRAVE \\
\hline $\begin{array}{l}\text { Si en la pregunta anterior ha contestado Sí: } \\
\text { ¿Cómo fueron las consecuencias del error? }\end{array}$ & $\square$ & $\square$ & $\square$ \\
\hline $\begin{array}{l}\text { ¿Necesitó ingreso hospitalario? } \\
\text { ¿Cuántos días estuvo ingresado/a? }\end{array}$ & & SÍ & NO \\
\hline
\end{tabular}

Si en la pregunta anterior ha contestado Sí:

\begin{tabular}{|l|c|c|c|}
\hline & MUY GRAVES & POCO GRAVES & NADA GRAVE \\
\hline ¿Cómo fueron las consecuencias del error? & $\square$ & $\square$ & $\square$ \\
\hline
\end{tabular}

¿Necesitó ingreso hospitalario?

¿Cuántos días estuvo ingresado/a?:

\begin{tabular}{|c|l|c|c|c|}
\hline \multicolumn{7}{|l|}{ 7. ¿Con qué probabilidad cree que alguna de estas cosas puede pasarle a usted? } \\
\hline a & Un accidente de trafico & ALTA & MEDIA & BAJA \\
\hline b & Un incendio por cortocircuito & & & \\
\hline c & Un atraco / un robo & & & \\
\hline d & Un error clínicocon consecuencias graves & & & \\
\hline e & Una infección alimentaría & & & \\
\hline
\end{tabular}

Por favor, indique:

$\begin{array}{lrrr}\text { Su edad: } & \text { años } \quad \text { Si es: Hombre } \square \quad \text { Mujer } \square & \\ \text { Estado civil: soltero/a } \square \quad \text { casado/a } \square \quad \text { viudo/a } \square & \text { divorciado/a } \square \\ \text { Estudios concluidos: básicos } \square \quad \text { form. profesional } \square & \text { universitarios } \square \\ \text { Años en tratamiento en diálisis: } & \text { años } & \end{array}$

\section{BIBLIOGRAFÍA}

1. Brennan ta, Leape Ll, Laird NM, Hebert L, LoCALIO AR, LAWTHERS AG, et al. Incidence of adverse events and negligence in hospitalized patients. Results of the Harvard Medical Practice Study I. N Engl J Med 1991; 324: 370376.

2. Wilson RM, Runciman WB, GibBerd RW, HaRrisson BT, Newby L, Hamilton JD. The quality in Australian Health-Care Study. Med J Aust 1995; 163: 458-471.

3. Healey M, Shackford S, Osler T, Rogers F, Burns E. Complications in surgical patients. Arch Surg 2002 ;137: 611-618.
4. Aranaz-Andrés JM, Aibar-Remón C, Vitaller-Burillo J, Gea-Velazquez de Castro MT, Requena-PuCHE J, TEROL-GARCíA E AND THE ENEAS WORKGROUP. Impact and Preventability of adverse events in Spanish public hospitals. Results of the ENEAS study JECH 2008; 62: 1022-1029.

5. Quori A, BaAmonde-Laborda E, García-Cantón C, Lago-Alonso MM, Toledo-González A, MonzónJIMÉNEZ E et al. Surveillance for infections and other adverse events in dialysis patients in southern Gran Canaria. Nefrologia 2011; 7: 457-463.

6. Rosser W, Dovey S, Bordman R, White D, CRighton E, DRummond N. Medical errors in primary care. Can Fam Physician 2005; 51: 386-387. 
7. Hickner J, Graham DG, Elder NC, Brandt E, Emsermann CB, Dovey $S$ et al. Testing process errors and their harms and consequences reported from family medicine practices: a study of the American Academy of Family Physicians National Research Network. Qual Saf Health Care 2008; 17: 194-200.

8. Garfield S, Barber N, Walley P, Willson A, EliasSON L. Quality of medication use in primary care-mapping the problem, working to a solution: a systematic review of the literature. BMC Medicine 2009; 7: 50.

9. Aranaz JM, Aibar C, Vitaller J, Mira JJ, Orozco D. Estudio APEAS: estudio sobre la seguridad de los pacientes en Atención Primaria de Salud. Ministerio de Sanidad y Consumo (España). Madrid 2008. [Consultado: 22 Agosto 2011]. Disponible en: http://www.msc.es.

10. Sarkar U, Handley MA, Gupta R, Tang A, Murphy E, SEligman HK et al. What happens between visits? Adverse and potential adverse events among a low-income, urban, ambulatory population with diabetes. Qual Saf Health Care 2010; 19: 223-228.

11. Massó P, Aranaz J, Mira JJ, Perdiguero E, Aibar C. Adverse Events in hospitals: The patient's point of view. Qual Saf Health Care 2010; 19: 144-147.

12. Evans SM, Berry JG, Smith BJ, Esterman AJ. Consumer perceptions of safety in hospitals. BMC Public Health 2006; 6: 41.

13. Mira JJ, Aranaz J, Vitaller J, Ziadi M, Lorenzo S, Rebasa $P$ et al. Percepción de seguridad clínica tras el alta hospitalaria. Med Clin (Barc) 2008; 131 (Supl. 3): 26-32.

14. King A, Daniels J, Lim J, Cochrane DD, TaYloR A, Ansermino JM. Time to listen: a review of methods to solicit patient reports of adverse events. Qual Saf Health Care 2010; 19: 148-157.

15. Mira JJ, Navarro I, Gullabert M, Aranaz J. Estudio de frecuencia de errores de los pacientes con su medicación. Revista Panamericana de Salud Pública/Pan American Journal of PublicHealth (en prensa).

16. Mira JJ, Nebot C, Lorenzo S, Pérez-Jover V. Patient report on information given, consultation time and safety in primary care. Qual Saf Health Care 2010; 19: e33 Epub 2010 May 27.

17. Adams RE, Boscarino JA. A community survey of medical errors in New York. Int $\mathrm{J}$ Qual Health Care 2004; 16: 353-362.
18. Weingart SN, Price J, Duncombe D, Connor M, Sommer K, Conley KA et al. Patient-reported safety and quality of care in outpatient oncology. Comm J Qual Patient Saf 2007; 33: 83-94.

19. Weissman JS, Scheneider EC, Weingart SN, Epstein AM, David-Kasdan J, Feilbelman $S$ et al. Comparing patient-reported hospital adverse events with medical record review: Do patients know something that hospitals do not? Ann Intern Med 2008; 149: 100-108.

20. Weingart SN, Pagovich O, Sands DZ, Li JM, AronSON MD, DAvis RB et al. What can hospitalized patients tell us about adverse events? Learning from patient-reported incidents. J Gen Intern Med 2005; 20: 830-836.

21. Aranaz-Andrés JM, Limón-Ramírez R, AibarRemón C, Miralles-Bueno JJ, Vitaller-Burillo J, Terol-García E et al. Luces y sombras en la seguridad del paciente: estudio y desarrollo de estrategias. Gac Sanit 2008; 22 (Supl. 1): 198-204.

22. Ministerio SAnidad y Consumo. Barómetro Sanitario. 2008. Disponible en: http://www.msc. es/estadEstudios/estadisticas/inforRecopilaciones/barometro/home.htm. [consultado 19 septiembre de 2010 ].

23. Mira JJ, Lorenzo S, Vitaller J, Ziadi M, Ortíz L, IgNACio E et al. El punto de vista de los pacientes sobre la seguridad clínica de los hospitales. Validación del Cuestionario de Percepción de Seguridad. Revista Médica de Chile 2009; 137; 1441-1448.

24. Alonso-Hernández P, Otero MJ, Maderuelo JA. Ingresos hospitalarios causados por medicamentos: incidencia, características y coste. Farmacia Hosp (Madrid) 2002; 26: 77-89.

25. Grissinger M, Lease M. Misadventures insulin theRAPY ARE YOU AT RISK? J National Medical Association 2003; 95 (Suppl. 1): 1S-16S.

26. UNESPA. Informe sobre riesgos al volante. Disponible en: http://www.unespa.es/frontend/unespa/Calcula-Tu-Riesgo-Al-Volantevn2868-vst244. [consultado 8 de septiembre 2011]

27. Blenkinsopp A, Wilkie P, Wang M, Routledge PA. Patient reporting of suspected adverse drug reactions: a review of published literature and international experience. Br J Clin Pharmacol 2006; 63: 148-156. 